\title{
Vacuum-Assisted Wound Closure with Mesh-Mediated Fascial Traction Achieves Better Outcomes than Vacuum-Assisted Wound Closure Alone: A Comparative Study
}

\author{
Giuseppe Salamone $^{1} \cdot$ Leo Licari $^{1} \cdot$ Giovanni Guercio $^{1} \cdot$ Albert Comelli $^{2}$ • \\ Mirko Mangiapane $^{1} \cdot$ Nicolò Falco $^{1} \cdot$ Roberta Tutino $^{1} \cdot$ Noemi Bagarella $^{1}$. \\ Sofia Campanella ${ }^{1}$ - Calogero Porrello ${ }^{1} \cdot$ Roberto Gullo $^{1} \cdot$ Gianfranco Cocorullo $^{1}$. \\ Gaspare Gulotta ${ }^{1}$
}

Published online: 16 November 2017

(C) The Author(s) 2017. This article is an open access publication

\begin{abstract}
Background Open abdomen (OA) permits the application of damage control surgery principles when abdominal trauma, sepsis, severe acute peritonitis and abdominal compartmental syndrome (ACS) occur.

Methods Non-traumatic patients treated with OA between January 2010 and December 2015 were identified in a prospective database, and the data collected were retrospectively reviewed. Patients' records were collected from charts and the surgical and intensive care unit (ICU) registries. The Acosta "modified" technique was used to achieve fascial closure in vacuum-assisted wound closure and mesh-mediated fascial traction (VAWCM) patients. Sex, age, simplified acute physiology score II (SAPS II), abdominal compartmental syndrome (ACS), cardiovascular disease (CVD) and surgical technique performed were evaluated in a multivariate analysis for mortality and fascial closure prediction.

Results Ninety-six patients with a median age of 69 (40-78) years were included in the study. Sixty-nine patients $(72 \%)$ underwent VAWCM. Forty-one patients $(68 \%)$ achieved primary fascia closure: two patients $(5 \%)$ were treated with VAWC (37 median days) versus 39 patients (95\%) who were treated with VAWCM (10 median days) $(p=0.0003)$. Forty-eight patients underwent OA treatment due to ACS, and 24 patients $(50 \%)$ survived compared to 36 patients $(75 \%)$ from the "other reasons" group $(p=0.01)$. The ACS group required longer mechanical ventilator support $(p=0.006)$, length of stay in hospital $(p=0.005)$ and in ICU $(p=0.04)$ and had higher SAPS II scores $(p=0.0002)$.

Conclusions The survival rate was $62 \%$. ACS $(p=0.01)$, SAPS II $(p=0.004)$, sex $(p=0.01)$, pre-existing CVD $(p=0.0007)$ and surgical technique (VAWC vs VAWCM) $(p=0.0009)$ were determined to be predictors of mortality. Primary fascial closure was obtained in $68 \%$ of cases. VAWCM was found to grant higher survival and primary fascial closure rate.
\end{abstract}

Leo Licari

lele.licari@gmail.com

1 General and Emergency Surgery - Policlinico P. Giaccone, University of Palermo, Via Liborio Giuffré 5, 90127 Palermo, Italy

2 Department of Industrial and Digital Innovation, Policlinico P. Giaccone, University of Palermo, Via Liborio Giuffré 5, 90127 Palermo, Italy

\section{Introduction}

Temporary abdominal closure (TAC) is the easiest way to facilitate re-operations when needed, such as in secondary and tertiary peritonitis or acute haemorrhagic necrotic pancreatitis or trauma.

Contemporary use of negative pressure therapy (NPT) allows the reduction in bacterial load and pro-inflammatory cytokines [1-6]. It also allows the standardisation of open 
abdomen (OA) techniques, improving prognosis and results of the procedure [6-9].

Recently, useful innovations in $\mathrm{OA}$ techniques have been introduced, and the indications are better defined to apply them in urgent situations as well as different surgical scenarios.

In 1897, McCosh was the first surgeon to describe an OA technique for the treatment of generalised peritonitis. He treated these cases, "leaving the abdomen opened, placing surgical sterile drapes amongst bowel and abdominal wall allowing the drainage of peritoneal exudate and peritoneal lavage" [10]. The proposed technique was first abandoned, probably due to the scepticism of scientists, and then revived later when general indications of OA were established by the introduction of the damage control surgery (DCS) concept and its further modifications (Fig. 1).

Novel indications in treatment of OA go beyond severe abdominal trauma, abdominal sepsis, severe acute peritonitis and other conditions that could evolve into abdominal compartmental syndrome (ACS) due to intraabdominal hypertension (IAH). The World Society of Abdominal Compartment Syndrome (WSACS) defines ACS as "sustained Intra-Abdominal Pressure (IAP)
$>20 \mathrm{mmHg}$ associated with new onset organ dysfunction" [3]. Mortality associated with ACS is estimated to be between 36 and $80 \%$, respectively, among patients with decompression versus untreated patients.

OA treatment requires the application of TAC.

Ideally, TAC should be easy to perform and rapidly reversible. It should prevent evisceration, preserve abdominal fascia, avoiding retraction of margins, prevent dehydration and adherence formation, drain peritoneal fluids, reduce bacterial load, reduce cytokine rate and permit easy fascia closure [11-20].

In 2007, Acosta et al. described the vacuum-assisted wound closure and mesh-mediated fascial traction technique (VAWCM). They temporarily sutured a polypropylene mesh to fascia medial margins until definitive abdominal closure was achieved according to general clinical/surgical conditions and IAP. In 2011, Acosta et al. published a prospective study in which they demonstrated that in $89 \%$ of cases treated with the VAWCM, late fascia closure was obtained with a median of 14 days of OA treatment.

This study aimed to assess survival and risk factors associated with mortality for non-traumatic patients treated with $\mathrm{OA}$ and evaluate the outcome in patients treated with

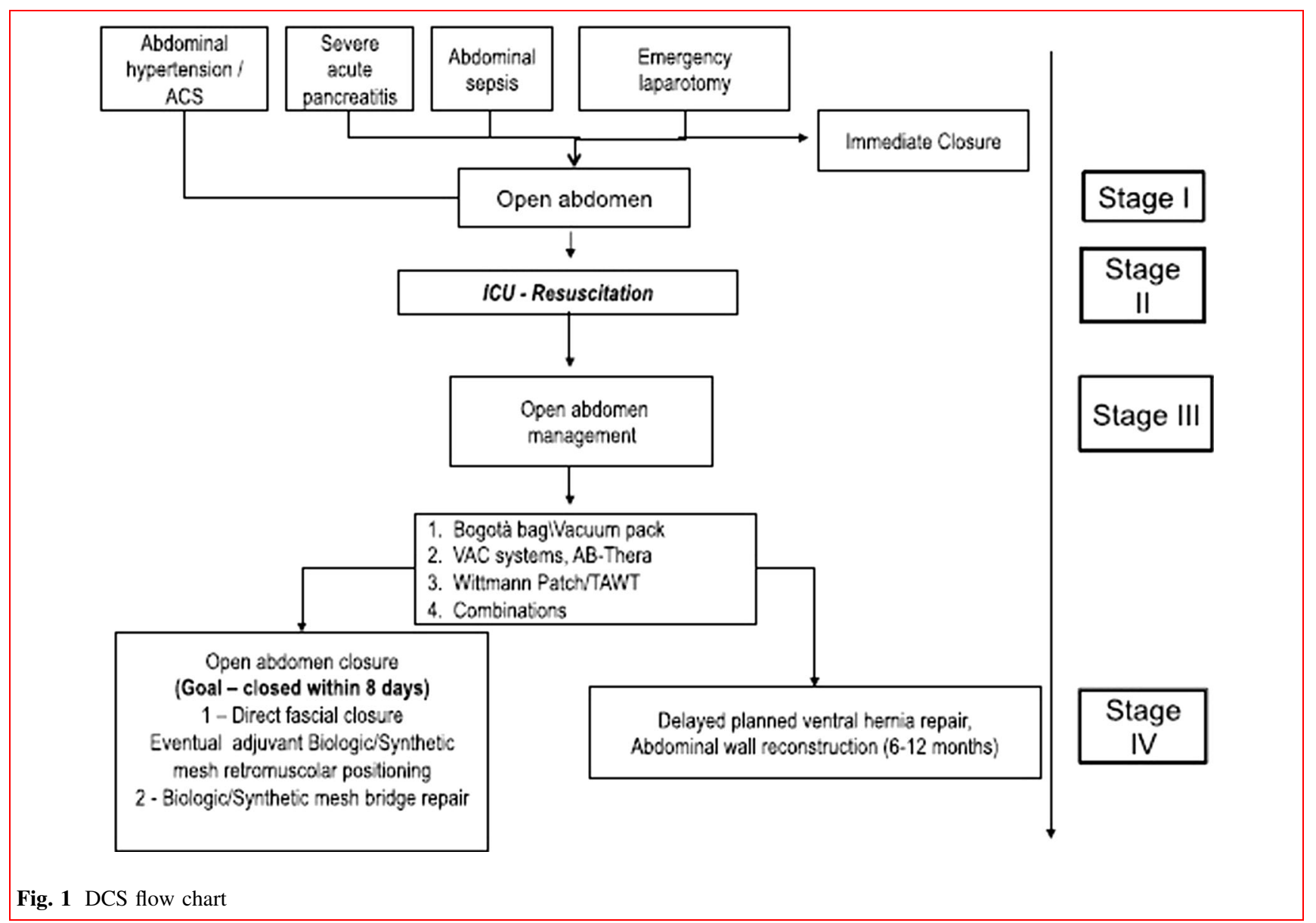


OA for ACS and evaluate the fascial closure rate, in-hospital and intensive care unit (ICU) length of stay (LOS) and time to abdominal closure in patients treated with VAWCM.

\section{Patients and methods}

Non-traumatic patients treated with $\mathrm{OA}$ at the Policlinico "Paolo Giaccone" at Palermo University Hospital between January 2010 and December 2015 were identified in a prospective database, and the data collected were retrospectively reviewed; patients' medical and surgical records were collected from charts and the surgical and ICU registries.

The simplified acute physiology score (SAPS II) was collected from the ICU registry and applied according to score indication.

IAP was measured with the intra-vesical technique using the Unometer $^{\mathrm{TM}}$ Abdo-pressure ${ }^{\mathrm{TM}}$ (ConvaTec Inc., Greensboro, USA) at least every $6 \mathrm{~h}$.

Diagnosed ACS was treated with surgical decompression. For some patients, OA treatment was performed to prevent ACS. For the treatment of patients with OA, Negative Pressure Suprasorb ${ }^{\circledR}$ CNP P1 (Lohmann \& Rauscher, Rengsdorf, Germany), with a continuous negative pressure between 25 and $125 \mathrm{mmHg}$, was applied. Dressing changes were performed every $48-72 \mathrm{~h}$ in the operating room. Patients were treated with VAWCM or negative pressure wound therapy (NPWT) with only vacuum-assisted wound closure (VAWC).

The fascia was closed at the end of the OA treatment with running sutures using glycolide and trimethylene carbonate. When fascia closure was impossible due to the fascia retraction or to loss of domain, a planned ventral hernia was performed. Classification of the OA was done according to Björck's classification.

To perform the VAWCM, the "modified" Acosta temporary abdominal closure (TAC) technique was adopted. The originally proposed technique involves a continuous running suture of polypropylene mesh along the entire length of the medial margins of the rectal fascia. Then, it is longitudinally cut and re-sutured, with a gradual overlap of the margins until the IAP, the oedema of the viscera and the general surgical conditions allow the fascial suture in the midline and the entire removal of the previously positioned prosthesis.

Our approach considers the opening of the anterior rectus sheath and the positioning of the polypropylene mesh inside the muscle cavity at approximately two centimetres under the muscle plane, fixed at the posterior layer. The mesh is positioned during the first dressing change, 48-72 $\mathrm{h}$ after the initial surgical operation. The medial margin is then sutured to reconstruct the integrity of the anterior rectus sheath. The mesh is thus longitudinally cut and re-sutured following the principles of the Acosta technique. At the end of the treatment, instead of the complete removal of the prosthesis, it is cut near the medial margin of the sheath, leaving a two-centimetre-wide strip anchored to the sheath. The strip increases the strength of the fascia in the midline, as support for the continuous running suture for abdominal wall reconstruction and closure.

The modified Acosta technique follows the principle of abdominal wall reconstruction with the use of polypropylene meshes positioned underneath even though they are medially fixed instead of laterally fixed in this case.

No short- and long-term complications were recorded due to the modified technique adopted.

\section{Statistics}

Data were analysed in Excel 2013 and IBM SPSS software, version 21 . The median was obtained for continuous variables. Comparison of continuous variables was made using Student's $t$ test or Mann-Whitney test, where appropriate. Comparison of categorical variables was made with the Chi-squared $\left(\chi^{2}\right)$ test or Fisher's exact test. The statistical significance level was set to $p$ value $<0.05$. Univariate analysis for survival was performed; the clinical variables included were age, SAPS II score, sex, ACS, cardiovascular disease (CVD) and mesh-mediated fascial closure. The variables with $p$ values $<0.05$ in univariate analysis were included in the multivariate logistic regression, considering odds ratios with $95 \%$ confidence intervals and $p$ values $<0.05$.

\section{Results}

Between January 2010 and December 2015, 96 patients were identified as receiving OA treatment. Sixty-six (69\%) were male, with a median age of 69 (40-78) years, and 48 (50\%) patients had ACS (Table 1). Sixty-nine patients (72\%) underwent VAWCM (Table 2). Bowel obstruction was the most common diagnosis (33\%), followed by necrotic haemorrhagic acute pancreatitis (19\%) (Table 3). Forty-one (42\%) patients were classified as grade 1A OA, $23(24 \%)$ as $1 \mathrm{~B}, 14(14 \%)$ as $1 \mathrm{C}, 10(10 \%)$ as $2 \mathrm{~A}, 5(5 \%)$ as $2 \mathrm{~B}, 3(3 \%)$ as $2 \mathrm{C}, 1(1 \%)$ as $3 \mathrm{~B}$ and $1(1 \%)$ as 4 .

Forty-eight patients had OA performed for ACS with IAP measured with median peak values of 30 (20-45) $\mathrm{mmHg}$. Types of organ failure present in the ACS patients were renal $(n=15)$, respiratory $(n=12)$, cardiovascular $(n=8)$, combined respiratory and renal failure $(n=8)$ and combined respiratory and cardiovascular failure 
Table 1 Patients characteristics

\begin{tabular}{lll}
\hline Age & Median (range) & $69(40-78)$ \\
Sex & M/F & $66 / 30(69 / 31 \%)$ \\
Comorbidity & Cv & $34(35 \%)$ \\
& Hypertension & $66(69 \%)$ \\
& Pulmonary & $18(19 \%)$ \\
& Malignant & $12(12 \%)$ \\
& Diabetes & $6(6 \%)$ \\
& Neurological disease & $4(4 \%)$ \\
& Liver failure & $4(4 \%)$ \\
Indications for & Renal failure & $4(4 \%)$ \\
OAT & Vascular disease & $12(12 \%)$ \\
& ACS & $48(50 \%)$ \\
& Prophylactic & $26(27 \%)$ \\
& 2nd look & $8(8 \%)$ \\
& Full-thickness dehiscence & $14(15 \%)$ \\
\hline
\end{tabular}

Table 2 Type of TAC

\begin{tabular}{ll}
\hline All cases & 96 \\
\hline VAWC & $27(28 \%)$ \\
VAWCM & $69(72 \%)$ \\
\hline
\end{tabular}

Table 3 Surgical diagnosis

\begin{tabular}{lr}
\hline All cases & 96 \\
\hline Pancreatitis & 18 \\
Bowel obstruction & 32 \\
Bowel perforation & 24 \\
Sepsis & 8 \\
Mesenteric ischaemia & 14 \\
\hline
\end{tabular}

$(n=5)$. The OA technique was performed at a median of $72(24-120) \mathrm{h}$ after the primary surgical operation due to the need for a second look $(n=8)$ and full-thickness wound dehiscence $(n=14)$. Twenty-six patients were left prophylactically open at the end of the primary surgical operation. Seventy-nine patients were treated with VAWCM, and 27 patients were treated with VAWC.

Forty-one (68\%) of the patients who survived OA achieved primary fascia closure: 5\% treated with VAWC (37 median days) versus 95\% treated with VAWCM (10 median days) $(p=0.0003)$. Nineteen $(32 \%)$ were left with a planned ventral incisional hernia (Table 4).

The median number of dressing changes before ending treatment with OA or death was $10(1-35)$. Patients with
Table 4 Outcome of patients with VAWC versus VAWCM treatment

\begin{tabular}{lllll}
\hline & All & VAWC & VAWCM & $p$ value \\
\hline$N$ & 96 & $27(28 \%)$ & $69(72 \%)$ & \\
Survival & $60(62 \%)$ & $10(37 \%)$ & $50(72 \%)$ & 0.0012 \\
Dead & $36(38 \%)$ & $17(63 \%)$ & $19(28 \%)$ & \\
Age & $69(40-78)$ & $70(40-78)$ & $55(40-78)$ & 0.2700 \\
Male & $66(69 \%)$ & $15(55 \%)$ & $51(74 \%)$ & 0.0810 \\
Female & $30(31 \%)$ & $12(45 \%)$ & $18(26 \%)$ & \\
Saps II & $40(14-82)$ & $50(19-82)$ & $35(14-77)$ & 0.0002 \\
Los in $H$ & $30(1-105)$ & $40(1-105)$ & $25(1-105)$ & 0.0063 \\
Los in ICU & $18(1-70)$ & $32(1-70)$ & $11(1-70)$ & 0.0520 \\
Days on ventilator & $15(1-60)$ & $21(1-50)$ & $7(1-60)$ & 0.0077 \\
Fascial closure rate & $41(68 \%)$ & $2(5 \%)$ & $39(95 \%)$ & 0.0003 \\
Incisional hernia & $19(32 \%)$ & $8(42 \%)$ & $11(58 \%)$ & \\
\hline
\end{tabular}

primary fascial closure could be closed after 30 (9-105) days, with a median of 82 (60-105) days for VAWC patients and 12 (9-35) days for VAWCM patients $(p=0.0025)$.

LOS in hospital was $30(1-105)$ days. LOS in ICU was 18 (1-70) days, which was required for all 96 patients with a median SAPS II score of 40 (14-82). The surviving group had a median SAPS II score of 37 (14-82) compared to 60 (20-82) among the non-survivors group $(p=0.0038)$. All patients required mechanical ventilation support with a median of 15 (1-60) days, of which 48 were in the ACS group with a median of 21 (1-50) days compared to 11 (1-60) days for the "other reasons for OAT" group $(p=0.006)$.

Sixty (62\%) patients survived to OA treatment with a primary fascial closure rate of $68 \%$. Non-survivors (38\%) had a median of $2(1-4)$ organ failures. Deaths were related to the organ failure, but no deaths were attributed to the OA technique. Forty-eight patients underwent $\mathrm{OA}$ treatment due to ACS, and 24 patients (50\%) survived compared to the $35(75 \%)$ of those that underwent OA for other reasons $(p=0.01)$. Nevertheless, the ACS group required longer mechanical ventilator support, LOS in hospital and in ICU and had higher SAPS II scores (Table 5).

In a univariate analysis of predictors for mortality, ACS, SAPS II, sex, pre-existing CVD and surgical technique (VAWC vs VAWCM) increased the risk of death (Table 6). In the multivariate logistic regression analysis, the same variables were demonstrated to be statistically significant predictors for in-hospital mortality as follows: SAPS II ( $p$ value 0.004 ; OR 0.97 ; 95\% CI 0.95-0.99); sex ( $p$ value 0.01 ; OR $0.27 ; 95 \%$ CI $0.09-0.8$ ); ACS ( $p$ value 0.013; OR 2.18; 95\% CI 0.79-6.04); pre-existing CVD 
Table 5 Outcome of patients due to the reasons of OA treatment

\begin{tabular}{|c|c|c|c|c|}
\hline & All & ACS & Other reasons & $p$ value \\
\hline$N$ & 96 & 48 & 48 & \\
\hline Survival & $60(62 \%)$ & $24(50 \%)$ & $36(75 \%)$ & 0.0100 \\
\hline Dead & $36(38 \%)$ & $24(50 \%)$ & $12(25 \%)$ & \\
\hline Age & $60(40-78)$ & $71(40-78)$ & $65(50-78)$ & 0.2600 \\
\hline Male & $66(69 \%)$ & $32(67 \%)$ & $34(71 \%)$ & 0.6600 \\
\hline Female & $30(31 \%)$ & $16(33 \%)$ & $14(29 \%)$ & \\
\hline Saps II & $40(14-82)$ & $49(19-82)$ & $32(14-77)$ & 0.0002 \\
\hline Los in $H$ & $30(1-105)$ & $32(1-105)$ & $27(1-105)$ & 0.0050 \\
\hline Los in ICU & $18(1-70)$ & $20(1-70)$ & $12(1-70)$ & 0.0470 \\
\hline Days on ventilator & $15(1-60)$ & $21(1-50)$ & $11(1-60)$ & 0.0060 \\
\hline
\end{tabular}

Table 6 Prediction of mortality

\begin{tabular}{|c|c|c|c|c|c|c|c|c|c|}
\hline & \multirow[t]{2}{*}{ All } & \multirow[t]{2}{*}{ Survived } & \multirow[t]{2}{*}{ Dead } & \multicolumn{3}{|c|}{ Univariate } & \multicolumn{3}{|c|}{ Multivariate } \\
\hline & & & & OR & $95 \% \mathrm{CI}$ & $p$ value & OR & $95 \% \mathrm{CI}$ & $p$ value \\
\hline$N$ & 96 & $60(62 \%)$ & $36(38 \%)$ & & & & & & \\
\hline Age & $69(40-78)$ & $65(40-78)$ & $72(47-78)$ & 0.9919 & $0.9593-1.0257$ & 0.636 & 1.0092 & $0.9729-1.0469$ & 0.623 \\
\hline Saps II & $40(14-82)$ & $37(14-82)$ & $60(20-82)$ & 0.9698 & $0.9498-0.9902$ & 0.003 & 0.9681 & $0.9469-0.9897$ & 0.004 \\
\hline Male & $66(69 \%)$ & $46(77 \%)$ & $20(55 \%)$ & 0.3804 & $0.1564-0.9253$ & 0.033 & 0.2713 & $0.0919-0.8013$ & 0.018 \\
\hline Female & $30(31 \%)$ & $14(23 \%)$ & $16(45 \%)$ & & & & & & \\
\hline ACS & $48(50 \%)$ & $24(40 \%)$ & $24(67 \%)$ & 3.0000 & $1.2641-7.1198$ & 0.012 & 2.1823 & $0.7887-6.0383$ & 0.013 \\
\hline Other reasons & $48(50 \%)$ & $36(60 \%)$ & $12(33 \%)$ & & & & & & \\
\hline $\mathrm{Cv}$ disease & $34(35 \%)$ & $14(23 \%)$ & $20(55 \%)$ & 4.1071 & $1.6886-9.9900$ & 0.001 & 6.6032 & $2.2207-19.6343$ & 0.0007 \\
\hline Other disease & $62(65 \%)$ & $46(77 \%)$ & $16(45 \%)$ & & & & & & \\
\hline VAWCM & $69(72 \%)$ & $50(83 \%)$ & $19(53 \%)$ & 0.2235 & $0.0871-0.5739$ & 0.001 & 0.1498 & $0.0486-0.4615$ & 0.0009 \\
\hline VAWC & $27(28 \%)$ & $10(17 \%)$ & $17(47 \%)$ & & & & & & \\
\hline
\end{tabular}

( $p$ value 0.0007 ; OR 6.60; 95\% CI 2.22-19.63); surgical technique (VAWC vs VAWCM) ( $p$ value 0.0009; OR 0.15; 95\% CI 0.05-0.46).

The Acosta modified technique we suggested showed the absence of short- and long-term complications such as SSI, seroma formation, evisceration, intra-abdominal abscesses, haemorrhages and sepsis, allowing the abdominal wall closure.

Incisional hernia was then recognised through physical examination and radiological findings (computed tomography $[\mathrm{CT}]$ scan).

\section{Discussion and conclusions}

The damage control surgery (DCS) concept was introduced in 1983 by Stone and then re-interpreted in 1993 by Rotondo and Scwhab and was first used to improve the prognosis of severe abdominal trauma [21, 22].

It is made up of three treatment stages: first, an urgent surgical operation is performed to find and control bleeding and/or infective sources through abdominal packing techniques, systematic peritoneal cavity exploration and lavages. Then, OA is performed to avoid ACS and to allow further re-operations, protecting abdominal fascia integrity. ICU transfer represents the fundamental phase of the treatment to obtain stabilisation and improvement of vital parameters. The last stage is the gold standard surgical procedure and the definitive fascia closure.

TAC represents an easy method of facilitating reoperations. 
In 2009, Koperna et al. evaluated the importance of performing a re-laparotomy in $48 \mathrm{~h}$ versus after $48 \mathrm{~h}$, finding that the mortality rate was 28 versus $76.5 \%$. This evidence demonstrates the relevance of the DCS and of the OA treatment instead of re-laparotomy on demand [15]. OA treatment with the postponement of definitive surgery allows a rapid surgical operation with the purpose of DCS. It allows systematic reviews of the abdominal cavity with repeated peritoneal lavages and drainage of abdominal secretions. This procedure can reduce bacterial load and cytokine rate and allows planned abdominal fascia closure, thereby avoiding ACS development. Bleszynski et al. evaluated the mortality risk between patients with a predicted mortality rate of $45 \%$ through APACHE-IV score treated with re-laparotomy on demand or OA. Data showed a mortality risk of 38.6 versus $22.8 \%$ [16-18].

In 2009, Björck et al. [23] proposed a classification of OA to standardise the treatment of abdominal sepsis. This classification was then reviewed in 2016 (Fig. 2). OA treatment requires the application of a TAC. Initial examples of TAC were the skin-only closure and the Bogotà bag. Both of these techniques are characterised by the ease and rapidity of their performance. They are also low-cost procedures. The most important disadvantage is the retraction of fascial margins.

A solution for fascia margin retraction was found between the late 1980s and the early 1990s with the appearance of meshes and the Wittmann Patch technique.

Finally, the vacuum pack seemed to solve the problem of abdominal secretions and their drainage. It consists of the placement of a polyethylene dressing in contact with the viscera, which is covered by surgical drapes and iodine dressings and connected to the vacuum system with a negative pressure of $100-150 \mathrm{mmHg}$. The evolution of this technique is the NPWT associated with the OA technique [24-26].
In 2012, Roberts et al. conducted a meta-analysis that compared 2 randomised controlled trials and 9 cohort studies ( 3 prospective and 6 retrospective). They demonstrated that the OA treatment with NPWT compared with other possible TAC has a lower mortality rate (18 vs $27 \%$ ), major late closing fascia rate (60 vs $52 \%)$, lower lactate serum levels and IAP and a shorter LOS.

In recent years, several alternative techniques have been published to optimise the OA technique and to prevent the retraction of fascia margins. In 2007, Acosta et al. described the VAWCM, which consists of the temporary suturing of a polypropylene mesh at fascia medial margins until abdominal definitive closure is permitted according to general clinical/surgical conditions and IAP. Subsequent dressing changes allow systematic review of the abdominal cavity according to NPWT and OA as well as the possibility to gradually accost the medial margins of the abdominal fascia. In 2011, Acosta et al. published a prospective study demonstrating that in $89 \%$ of cases treated with the VAWCM, late fascia closure was obtained with a median of 14 days of OA treatment. Furthermore, fewer re-explorations and a shorter duration of open abdominal management are associated with higher fascial closure rates [27].

In 2005, Miller et al. [28] demonstrated that prolonging the OA until the 8th to 9th postoperative day increases the probability of developing complications to nearly $25 \%$. The primary complications described are ACS, SSI and entero-atmospheric fistula (EAF). The latter is considered the worst complication that occurred frequently in severe sepsis syndrome [29-34].

The present study confirms that OA is the gold standard technique for the application of DCS principles. The observed survival rate was $62 \%$, which is similar to other results observed in scientific literature: Acosta et al. [35] reported a survival rate of $70 \%$, Carlson et al. [36] reported

\begin{tabular}{|c|c|c|c|}
\hline \multicolumn{2}{|c|}{2009 classification system } & \multicolumn{2}{|c|}{ Amended classification system } \\
\hline IA & $\begin{array}{l}\text { Clean OA without adherence between bowel and abdominal } \\
\text { wall or fixity (lateralization of the abdominal wall) }\end{array}$ & $1 \mathrm{~A}$ & Clean, no fixation \\
\hline \multirow{2}{*}{ IB } & Contaminated OA without adherence/fixity & $1 \mathrm{~B}$ & Contaminated, no fixation \\
\hline & & $1 C$ & Enteric leak, no fixation \\
\hline $2 \mathrm{~A}$ & Clean OA developing adherence/fixity & $2 \mathrm{~A}$ & Clean, developing fixation \\
\hline \multirow[t]{2}{*}{$2 \mathrm{~B}$} & Contaminated OA developing adherence/fixity & $2 \mathrm{~B}$ & Contaminated, developing fixation \\
\hline & & $2 \mathrm{C}$ & Enteric leak, developing fixation \\
\hline \multirow[t]{2}{*}{3} & OA complicated by fistula formation & $3 \mathrm{~A}$ & Clean, frozen abdomen \\
\hline & & 3B & Contaminated, frozen abdomen \\
\hline 4 & $\begin{array}{l}\text { Frozen OA with adherent/ fixed bowel, unable to close } \\
\text { surgically, with or without fistula }\end{array}$ & 4 & Established enteroatmospheric fistula, frozen abdomen \\
\hline
\end{tabular}

OA: open abdomen.

Enteric leak describes the situation where there is spillage of enteric contents into the abdomen without established enteric fistula development. An enteric leak controlled by closure, exteriorization into a stoma, or permanent enterocutaneous fistula is considered clean (Grades 1 and 2).

Fig. 2 Björck classification 
a rate of $72 \%$, and Seternes et al. [11] reported a rate of $68 \%$.

ACS, SAPS II, sex, pre-existing CVD and surgical technique (VAWC vs VAWCM) increased the risk of death. In the multivariate logistic regression analysis, the same variables were demonstrated to be statistically significant predictors for in-hospital mortality.

The reason for the use of the OA technique was ACS in $50 \%$ of cases, with a survival rate of $50 \%$, confirming that ACS is a life-threatening condition.

Primary fascial closure was obtained in $68 \%$ of cases, and only $32 \%$ required a planned incisional hernia. Roberts et al. adopted NPWT as the gold standard procedure.

The surgical technique described above has been demonstrated to have high potential for widespread adoption when VAWCM is required. It summarises the Acosta principles regarding the performance of mesh-mediated fascial traction in order to avoid the retraction of fascial margins. It also follows the principles of abdominal wall reconstruction by the positioning of a polypropylene mesh underlay. The mesh increases the strength of the fascia in the midline, providing support for the continuous running suture for abdominal wall reconstruction and closure. No short- and long-term complications were described in the series proposed with the highest closure rate, 95 versus $5 \%$ of patients with VAWC, and with lower median days for primary fascial closure, a higher survival rate and a shorter LOS in hospital and ICU.

In addition to the results obtained by the applied indication of the OA technique and the chosen TAC strategy, several other aspects influence patient outcomes. Management of severe sepsis and septic shock requires a multidisciplinary team to assess resuscitation, respiratory support and infection control.

\section{Compliance with ethical standards}

Conflict of interest All authors are declare that they have no conflict of interest.

Open Access This article is distributed under the terms of the Creative Commons Attribution 4.0 International License (http://crea tivecommons.org/licenses/by/4.0/), which permits unrestricted use, distribution, and reproduction in any medium, provided you give appropriate credit to the original author(s) and the source, provide a link to the Creative Commons license, and indicate if changes were made.

\section{References}

1. Balogh ZJ, Lumsdaine W, Moore E et al (2014) Postinjury abdominal compartment syndrome: from recognition to prevention. Lancet 384(9952):1466-1475
2. Malbrain ML, Cheatham ML, Kirkpatrick A et al (2006) Results from the international consensus of experts on intra-abdominal hypertension and abdominal compartment syndrome. Intensive Care Med 32:1722-1732

3. Kirkpatrick AW, Roberts DJ, De Waele J et al (2013) Intraabdominal hypertension and the abdominal compartment syndrome: updated consensus definitions and clinical practice guidelines from the World Society of the Abdominal Compartment Syndrome. Intensive Care Med 39:1190-1206

4. Sugrue M, Jones F, Janjua KJ et al (1998) Temporary abdominal closure: a prospective evaluation of its effects on renal and respiratory physiology. J Trauma 45:914-921

5. Bosscha K, Hulstaert PF, Hennipman A et al (1998) Fulminant acute pancreatitis and infected necrosis: results of open management of the abdomen and "planned" reoperations. J Am Coll Surg 187:255-262

6. Coccolini F, Biffl W, Catena F et al (2015) The open abdomen, indications, management and definitive closure. World J Emerg Surg 10:32

7. Lindstedt S, Malmsjö M, Hlebowicz J et al (2013) Comparative study of the microvascular blood flow in the intestinal wall, wound contraction and fluid evacuation during negative pressure wound therapy in laparostomy using the V.A.C. abdominal dressing and the ABThera open abdomen negative pressure therapy system. Int Wound J. https://doi.org/10.1111/iwj.12056

8. Frazee RC, Abernathy SW, Jupiter DC et al (2013) Are commercial negative pressure systems worth the cost in open abdomen management? J Am Coll Surg 216(4):730-733

9. Cheatham ML, Demetrides D, Fabian TC et al (2013) Prospective study examining clinical outcomes associated with negative pressure wound therapy system and Barker's vacuum packing technique. World J Surg 37:2018-2030. https://doi.org/10.1007/ s00268-013-2080-z

10. McCosh AJ II (1897) The treatment of general septic peritonitis. Ann Surg 25:687-697

11. Seternes A, Rekstad LC, Mo S et al (2017) Open abdomen treated with negative pressure wound therapy: indications, management and survival. World J Surg 41:152-161. https://doi.org/10.1007/ s00268-016-3694-8

12. Sartelli M, Abu-Zidan FM, Ansaloni L et al (2015) The role of the open abdomen procedure in managing severe abdominal sepsis: WSES position paper. World J Emerg Surg 10:35

13. Yuan Y, Ren J, He Y (2013) Current status of the open abdomen treatment for intra-abdominal infection. Gastroenterol Res Pract 2013:532013

14. Bleszynski MS, Chan T, Buczkowski AK (2016) Open abdomen with negative pressure device vs primary abdominal closure for the management of surgical abdominal sepsis: a retrospective review. Am J Surg 211:926-932

15. Koperna T, Schulz F (2000) Relaparotomy in peritonitis: prognosis and treatment of patients with persisting intraabdominal infection. World J Surg 24(1):32-37. https://doi.org/10.1007/ s002689910007

16. Sartelli M, Catena F, Di Saverio S et al (2014) Current concept of abdominal sepsis: WSES position paper. J World J Emerg Surg 9(1):22

17. Van Ruler O, Kiewiet JJ, Boer KR et al (2011) Failure of available scoring systems to predict ongoing infection in patients with abdominal sepsis after their initial emergency laparotomy. BMC Surg 11:38

18. Van Ruler O, Lamme B, de Vos R et al (2008) Decision making for relaparotomy in secondary peritonitis. Dig Surg 25(5):339-346

19. Björck M, Bruhin A, Cheatham M et al (2009) Classificationimportant step to improve management of patients with an open 
abdomen. World J Surg 33(6):1154-1157. https://doi.org/10. 1007/s00268-009-9996-3

20. Schein M, Saadia R, Jamieson JR et al (1986) The 'sandwich technique' in the management of the open abdomen. Br J Surg 73(5):369-370

21. Stone HH, Strom PR, Mullins RJ (1983) Management of the major coagulopathy with onset during laparotomy. Ann Surg 197(5):532-535

22. Rotondo MF, Schwab CF, McGonigal MD (1993) 'Damage control': an approach for improved survival in exsanguinating penetrating abdominal injury. J Trauma 35(3):375-382

23. Björck M, Kirkpatrick AW, Cheatham M et al (2016) Amended classification of the open abdomen. Scand J Surg 105(1):5-10. https://doi.org/10.1177/1457496916631853 Epub 2016 Feb 29

24. Saxena V, Hwang CW, Huang S et al (2004) Vacuum-assisted closure: microdeformations of wounds and cell proliferation. Plast Reconstr Surg 114(5):1086-1096

25. Chen SZ, Li J, Li XY et al (2005) Effects of vacuum-assisted closure on wound microcirculation: an experimental study. Asian J Surg 28(3):211-217

26. Argenta LC, Morykwas MJ (1997) Vacuum-assisted closure: a new method for wound control and treatment: clinical. Ann Plast Surg 38(6):563-576

27. Atema JJ, Gans SL, Boermeester MA (2015) Systematic review and meta-analysis of the open abdomen and temporary abdominal closure techniques in non-trauma patients. World $\mathbf{J}$ Surg 39:912-925. https://doi.org/10.1007/s00268-014-2883-6
28. Miller RS, Morris JA Jr., Diaz JJ Jr. et al (2005) Complications after 344 damage-control open celiotomies. J Trauma 59(6):1365-1371

29. Bruhin A, Ferreira F, Chariker M et al (2014) Systematic review and evidence based recommendations for the use of negative pressure wound therapy in the open abdomen. Int J Surg 12(10):1105-1114

30. Layton B, DuBose J, Nichols S et al (2010) Pacifying the open abdomen with concomitant intestinal fistula: a novel approach. Am J Surg 199:e48-e50

31. Di Saverio S, Villani S, Biscardi A et al (2011) Open abdomen with concomitant enteroatmospheric fistula: validation, refinements, and adjuncts to a novelapproach. J Trauma 71(3):760-762

32. Losanoff JE, Richman BW, Jones JW (2003) Intestinal fistulization in the open treatment of peritonitis. Am J Surg 185:394

33. Mastboom WJ, Kuypers HH, Schoots FJ et al (1989) Small-bowel perforation complicating the open treatment of generalized peritonitis. Arch Surg 124:689-692

34. Kaushal M, Carlson GL (2004) Management of enterocutaneous fistulas. Clin Colon Rectal Surg 17(2):79-88

35. Acosta S, Bjarnason T, Petersson U et al (2011) Multicentre prospective study of fascial closure rate after open abdomen with vacuum and mesh-mediated fascial traction. $\mathrm{Br} \mathrm{J}$ Surg 98(735-74):3

36. Carlson GL, Patrick H, Amin AI et al (2013) Management of the open abdomen: a national study of clinical outcome and safety of negative pressure wound therapy. Ann Surg 257(1154-115):9 